\title{
Simulation of the PPARa regulome predicts PPARa activation exacerbates the development of hepatic steatosis
}

\author{
C.P. Fisher ${ }^{1}$, M. Khoury ${ }^{1}$, A.L. Barber ${ }^{1}$, D.J. Mazzatti ${ }^{2}$, N.J. Plant ${ }^{1}$, A.M. Kierzek ${ }^{1}$ and \\ J.B. Moore ${ }^{1}$ \\ ${ }^{1}$ School of Biosciences and Medicine, University of Surrey, UK and ${ }^{2}$ Unilever, Trumbell, Connecticut, USA
}

Non-alcoholic fatty liver disease (NAFLD) is a serious public health issue associated with obesity and the western diet. The molecular mechanisms mediating both the initial development of steatosis and its progression to steatohepatitis, and potentially fatal fibrosis and cirrhosis, are only partially understood. Utilizing a systems biology approach, the aim of this work was to identify key regulatory mechanisms involved in the molecular pathogenesis of NAFLD.

Beginning with comprehensive pathway analyses of our transcriptomic and proteomic datasets, generated from both in vitro and in vivo models of hepatocyte lipid loading and NAFLD, we identified perioxisome proliferator activated receptor alpha (PPAR $\alpha)$ as a key regulatory factor of hepatic lipid accumulation. Then, applying our novel quasi-steady state Petri nets (QSSPN) method ${ }^{1}$, we reconstructed an in silico model of the PPAR $\alpha$ regulome that is, to our knowledge, the most comprehensive dynamic model of PPAR $\alpha$ regulated hepatic metabolism. Using this model we simulated responses to increased fatty acid levels mimicking lipid-loading in vitro. These simulations predicted that, contrary to current thinking, PPAR $\alpha$ activation increases the initial steatotic response rather than mitigating against hepatic steatosis (Fig $1 \mathrm{a}, \mathrm{b}$ and $\mathrm{c}$ ). We went on to test this hypothesis experimentally using the PPAR $\alpha$ antagonist GW6471 and confirmed that activation of PPAR $\alpha$ does increase the initial rate of lipid accumulation in hepatocytes (Fig 1 d).
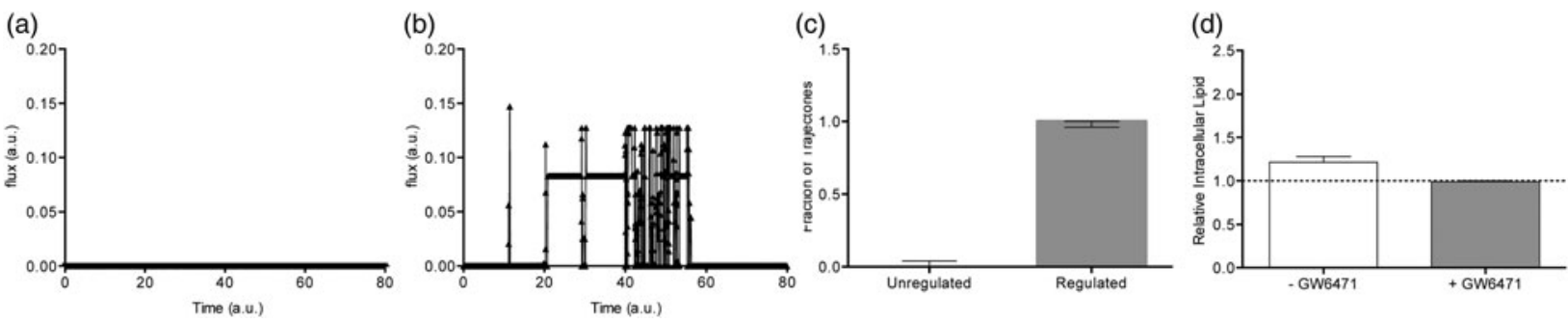

Fig 1: Simulation of final flux in triacylglycerol synthesis in response to oleic acid treatment using the reconstructed PPAR $\alpha$ regulome model. Simulated single trajectories in unregulated a) and regulated models b); c) fraction of trajectories showing increased flux towards triacyglycerol synthesis sampling 100 trajectories, data shown as fraction of trajectories \pm binomial probability confidence intervals. d) Relative intracellular lipid as quantified by nile red fluorescence in HepG2 cells treated with $400 \mu \mathrm{M}$ oleic acid \pm PPAR $\alpha$ antagonist GW6471 for 2 hrs, data shown as mean \pm SEM $(\mathrm{n}=4) ; \mathrm{P}=0.0363$.

We conclude, that by increasing the rate at which lipid accumulates in the initial stages of the hepatic response, PPAR $\alpha$ potentiates the damage of a high fat load to the liver. This would explain the lack of success in using PPAR $\alpha$ agonists as effective therapies for NAFLD and emerging evidence in the literature that although PPAR $\alpha$ agonists, such as fenofibrate, may reduce systemic hyerlipidemia, they exacerbate hepatic steatosis.

1. Fisher CP, Plant NJ, Moore JB \& Kierzek AM. (2013) QSSPN: Dynamic simulation of molecular interaction networks describing gene regulation, signalling and whole-cell metabolism in human cells. Bioinformatics 29(24): 3181-90. 